
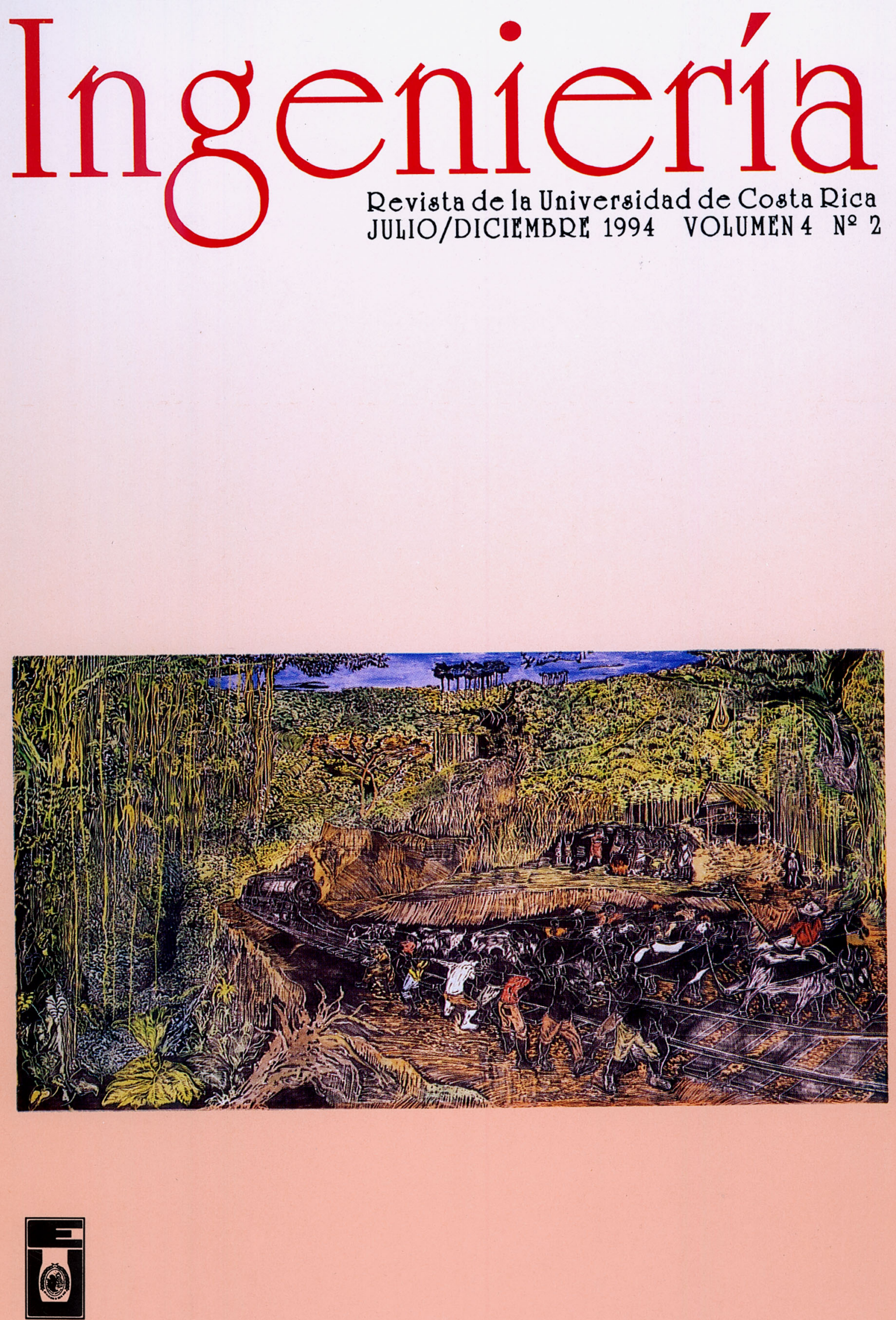


\section{LAS REDES DE COMUNICACIONES COMPUTARIZADAS PARA LA UNIVERSIDAD DEL SIGLO XXI}

RESUMEN
El desarrollo de redes computarizadas de comunicación constituye un proceso de formación de infraestructura, el cual, de acuerdo con enfoques económicos actuales, es un elemento clave para mejorar la competitividad de las organizaciones que las utilicen inteligemente.

Diversos países han asumido la tarea de convertir tal desarrollo en una estrategia nacional para mejorar los servicios - y las actividades de investigación, así como las transacciones comerciales globales.

La red INTERNET constituye el esfuerzo más amplio y ambicioso de contruir una red mundial de comunicaciones, que opera ya en más de 40 países y tiene cerca de 20 millones de usuarios, de las áreas académica, comercial y gubernamental.

En 1991, la Universidad de Costa Rica estableció el primer nodo nacional de la red BITNET, esfuerzo que luego dio origen a la rama nacional de Internet. Esta Universidad está en la etapa de construcción de una red corporativa innovadora, llamada internacionalmente UCRNet, basada en un anillo de comunicaciones de fibra óptica. El desarrollo de la rama académica de la red Internet nacional, CRNet,está dirigido por la Asociación CRNet. También están en su etapa inicial de construcción sus ramas comercial y gubernamental.

Es importante que para la consolidación nacional de Internet, los usuarios participen directamente en su desarrollo y administración.

\section{SUMMARY}

Computerized communication networks constitute a social effort to develop infrastructure which, according to present economic approaches, it's a means to form an strategic element for organizational competitiveness.

Sorne countries have established, as a national strategy, the development of such network.s in order to improve services and research activities, as well as global commercial transactions.

INTERNET is the most ambicious effort to build a world wide computerized communications network, formed by more than 40 national networks and almost 20 million users, from academic, commercial and governmental areas.

En 1991, the University ofCosta Rica established the first national node ofBITNET, effort that gave birth to the national Internet. This University. is building an innovative corporate network, called internationally as UCRNet, based on a fiber optics backbone. The development ot the academic branch oflnternet, CRNet, is directed by the CRNet Association. The commercial and governmental branch are also under construction.

It's important to note that users of the local branches of Internet must participate in their development and administration.

\section{LA TRANSNACIONALIZACION LAS ECONOMIAS}

Las crisis políticas en la actualidad son una de las expresiones de los cambios globales, o cambios de "paradigma" en términos de Kuhn. La separación global realmente importante no es más aquella que se da entre los países de economías de libre mercado y los de economías centralmente planificadas, sino la que existe entre sociedades pobres y sociedades ricas.

La división mencionada se evidencia primorialmente en lacapacidad de cada sociedad para desarrollar, transferir, adaptar, y disfrutar los productos de la aplicación de conocimientos científicos y tecnológicos. La "transnacionalización" de las problemáticas sociales y de los fenómenos económicos ponen de relieve los factores sobre los que actualmente se basan las relaciones de poder entre las naciones.

La relación entre ciencia, tecnología y desarrollo es de importancia primordial para las economías nacionales. Investigación e innovación constituyen palabras clave para el desarrollo económico. Dentro de las áreas de conocimiento, las Tecnologías de la Información (TI) parecen jugar un papel de pivote en tales cambios.

\footnotetext{
- Director, Centro de Informática, y Profesor, Escuela de Ciencias de la Computación e Informática .Universidad de Costa Rica
} 


\section{DESARROLLO Y GLOBALIDAD}

Hasta hace poco se consideraba que las ventajas competitivas de las naciones estaban basadas en sus ventajas comparativas nacidas de los factores productivos: trabajo, recursos naturales y costos de capital. Las economías de escala dictaban la regla "lo más grande es mejor" para triunfar en los mercados mundiales.

Actualmente, el trabajo de economistas e investigadores como Michael Porter de la Universidad de Harvard, mencionan que al menos dos factores hacen obsoleto tal enfoque: primero, la globalización de los mercados hace posible trasladar insumos desde o hacia cualquier lugar del mundo, donde el precio o la calidad sean más convenientes; y la capacidad de la ciencia y la tecnología para hac $\sim$ r po.sible la creación de ventajas competitivas a través de la innovación. De esos dos factores, la innovación tecnológica es el mayor agente de cambio! .

La inclusión en la Ronda de Uruguay del GA TI, de los temas: comercio de servicios, inversiones extranjeras directas y derechos de propiedad intelectual (introducido por Japón y los EUA en 1987), refleja la tendencia global de considerar a la ciencia y la tecnología como los elementos clave en la creación de ventajas comparativas para la competitividadmundial ${ }^{2}$, y la necesidad de controlar la producción de conocimientos nuevos.

Algunos países, como Japón o Singapur, con escasos recursos naturales, han sido ejemplo del desarrollo de ventajas competitivas "hechas por el hombre", a través de la aplicación del conocimiento y la creatividad. Así, el desarrollo se basa principalmente en las capacidades de las personas, localizadas en una área geográfica del mund $0^{3}$.

La competitividad está por tanto enraizada en los valores culturales, las características históricas, y las condiciones del contexto de un ambiente social específico. Dentro de este enfoque, actividades como educación, investigación y desarrollo productivo son componentes estratégicos para la competitividad de un país.

Al menos en los siguientes tres sectores están localizados los protagonistas principales de las actividades para el desarrollo de un país: empresarial, universitario y gubernamental. A estos se debe agregar el sector financiero.

\section{TECNOLOGIAS DE LA INFORMA- CION (TI) y DESARROLLO}

Las Tecnologías de la Información (TI) son un campo científico-tecnológico formado por la conjunción de al menos dos campos del conocimiento: la informática y las telecomunicaciones, cuyo objeto de estudio es el procesamiento, almacenamiento y transmisión de datos e información.

Las TI juegan un papel de pivote en los patrones de desarrollo del mundo presente. Ejemplo de áreas afectadas por las TI son las siguientes:

- Ganancias de productividad en algunos tipos de procesos e industrias. Ejemplos: diseño y construcción de vehículos, manufactura de computadoras, automatización de procesos administrativos.

- Mejoras en la calidad de procesos y productos. Ejemplos: construcción de automóviles, diseños publicitarios, diseño de circuitos impresos, mercadeo y distribución de productos.

- Ventajas directas para competir. Ejemplos: control de canales de distribución, sistemas de reservaciones de vuelo, distribución de listas, tarjetas de crédito. 
- Creación de nuevos procesos y productos. Ejemplos: técnicas de reconocimiento biométrico, robotización, ATMs, reconocimiento de patrones, libros electrónicos.

- Transformación de áreas de negocios, debido a la dificultad de asignarles fronteras tradicionales. Ejemplos: bancos, venta de vehículos y negocios detallistas.

Las TI también han marcado cambios profundos en la percepción que tienen los seres humanos de si mismos y su papel el mundo. Aun en los cambios políticos mundiales las TI han servido como un instrumento poderoso de transmisión de valores, patrones de consumo y aspiraciones de libertad.

\section{REDES DE COMUNICACION COMPU- TARIZADAS y COMPETITIVIDAD}

Países como Francia, Japón o Singapur han reconocido el valor estratégico de las TI. Por ejemplo, las capacidades del sistema de redes de comunicaciones computarizadas, PorNet, le han dado a Singapur una ventaja competitiva mundial de primera cIase en este campo.

Algunos gobiernos han asignado una prioridad alta a la inversión en Tecnologías de la Información. El actual vicepresidente de los Estados Unidos de América, EUA, Albert Gore, lideró la propuesta de establecer una red nacional de investigación y enseftanza, la "National Research and Education Network Program", NREN.

El presente Gobierno del presidente William Clinton a puesto en la arena político - económica la construcción de una "National Information Infrastructure", NII, o "super - highway" para trasmisión de datos, como una forma de construir una infraestructura que le de a ese país ventajas competitivas a nivel global.

La infraestructura tecnológica consiste de "ciencia, ingeniería, y conocimiento técnico disponible ...". Esta infraestructura no puede mejorar las actividades de investigación y desarrollo, y llegar hasta las transacciones comerciales globales. Por ejemplo, existe evidencia que la infraestructura de telecomunicaciones está causando crecimiento económico en años recientes".

La combinación del poder distribuido de las redes de comunicaciones computarizadas, debido principalmente al creciente número de microcomputadoras y estaciones de trabajo, y a las capacidades de las telecomunicaciones, han creado nuevas características sinergéticas a través de las redes de comunicaciones. Las redes de computadoras hacen al mundo más pequeño, decrecen la importancia de las barreras y distancias geográficas y del tiempo para la comunicación humana. Así, por ejemplo, las empresas de comercio financiero usan eficientemente las redes y sistemas de comunicación, tales como Nasdaq, Reuters, o Swift, 24 horas al día durante todo el año, a lo ancho y largo del planeta.

La operación de redes de comunicación computarizadas han traído nuevos y mejores servicios, tales como:

- Servicios universales: el acceso a una base común de servicios, en cualquier momento y lugar del planeta. Por ejemplo: redes financieras (Citicorp), o redes académicas (Internet).

- Bases de datos en línea: Información permanentemente accesible, en múltiples campos, tales como: negocios, agricultura, finanzas, noticias, etc. Esta información almacenada en computadoras, sería extremadamente difícil de obtener en otra formas debido al tiempo y costo asociados.

- Comercialización programada: comercialización de productos y su nexo con mercados financieros internacionales. Por ejemplo: las redes de comunicación hacen que los precios de productos agrícolas se alteren rápido, siguiendo otros cambios alrededor del mundo. La coordinación de estas actividades solo es posible con la ayuda de las TI. 
- Intercambio Electrónico de Datos ("EDI"): una estrategia para incrementar la calidad de los canales de comercialización entre empresas y suplidores.

Entre los cambios que las redes de comunicación han traído, se ecuentran también importantes problemáticas sociales y comerciales, las cuales no están resueltas y deben ser tratadas en su conjunto por la sociedad.

Entre tales problemáticas se encuentran las siguientes:

- Derechos de propiedad intelectual de los contenidos de la información que viaja en estas redes.

- Conflictos en la priorización de la inversión de infraestructura frente a otras necesidades sociales.

- Políticas de cobro y promoción de la demanda en el uso de estas redes.

- Derechos de privacidad para las personas.

- Seguridad de mensajes y documentos que viajan en las redes.

\section{LAS REDES INTERNET Y NREN}

La red de redes Internet es actualmente la más amplia red de computadoras del mundo. Opera en 40 o más países. El número de usuarios se estima en cerca de 20 millones, los cuales usan más de 1 millón de computadoras en alrededor de 7.500 redes interconectadas a través de todo el mundo. La comunidad de usuarios en EUA está facilmente entre entre 1 y 2 millones, y tiene el $80 \%$ de las computadoras $^{5}$ que la componen.

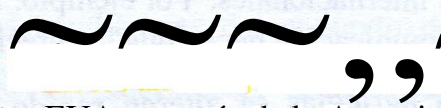

los EUA, a través de la Agencia para Proyectos de
Investigación Avanzados en Defensa (DARPA), apoyó el desarrollo de ARPANET, como un medio de comunicación y de compartir recursos entre investigadores. La "National Science Foundation", NSF, administra el núcleo, "backbone", de esa red, llamado NSFNET para incluir instituciones académicas en todo el país. Hoy, el tráfico a través de la NSFNET crece a un ritmo exponencial del $10 \%$ por mes.

Redes regionales y corporativas, como NEARNET de Nueva Inglaterra, son administradas directamente por organizaciones independientes. No hay un cuerpo rector central para toda la Internet. La NSF contrata el mantenimiento del backbone con Advanced Networking Services/Commercial, Research, and Education Inc., ANC/CORE, la cual es ahora el mayor proveedor de tráfico en Internet. ANC fue formada como un proyecto conjunto entre IBM, MCI y Merit ${ }^{6}$.

Solo en Australia, Canadá, el Reino Unido y EUA, sus gobiernos financian las actividades de Internet. En Europa, las restricciones comerciales a su uso son la excepción ${ }^{7}$. Una red tan exitosa como Bitnet nunca recibió fondos federales del gobierno de los EUA ${ }^{8}$. En Latinoamérica, por ejemplo, el Banco del Pacífico en Ecuador, ha financiado la infraestructura de Internet y vende servicios de conección a usuarios comerciales.

En el verano de 1991, EUA levantó las restricciones al uso comercial de Internet y, por ejemplo, en 1993 los siguientes eran los patrones de crecimiento en usuarios, destacándose el aumento en uso comercial:

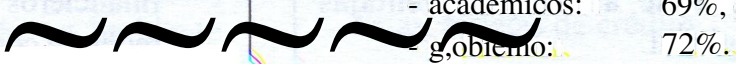


Aún muchas importantes empresas no se unen a Internet debido a preocupaciones sobre sus niveles de seguridad.

La administración de Internet se basa en un soporte fuerte de sus usuarios. La "Internet Society" y el "Federal Networking Council" asumen un nivel general de coordinación para la red de los EUA. La "Internet Engineering Task Force", IETF, es el cuerpo que establece las normas en Internet, y se divide en varios grupos de trabajo, de acuerdo con áreas técnicas. Sin embargo, Internet no ofrece aún servicios demandados por sus usuarios, tales como servicios de guía. El "Cornrnercial Internet Exchange", CIX, es un grupo de proveedores de redes privadas, formado hace unos dos años, para apoyar el uso comercial de Internet ${ }^{9}$.

Internet usa el "Transmission Control ProtocoVInternet Protocol", TCP/IP, para ligar a las subredes.

Entre los servicios de Internet se encuentran: el File Transfer Protocol de emulación, o Telnet, usado para apoyar el acceso a software a través de ejecución remota ("remote loggin "). Archie es un protocolo común para el acceso indizado de archivos FTP. Gopher, un sistema de navegación basado en menús para acceder computadoras remotas. Usenet, para foros públicos de discusión; etc.

El NREN es una red de alcance nacional para los EUA, propuesta en la "High PerformanceComputingAct" de 1991 (P.L.I02-194), para proveer "conexión entre instituciones de investigación y de educación, gobierno y empresas en todos los estados"1O. Está conexión será una infraestructura física con capacidad de banda altamente ancha (gigabits).

9 Office (1992), p. 17.

10 Kahin (1993): 102 P.L. 194. Section 102 (a), p. 407.

11 Office (1992), p. 2.

12 Porter(1990), pp. 617-618.
El Congreso de los EUA ha definido un programa de administración, formado por varios comités de diversas agencias para definir las necesidades de la futura NREN.

La NREN será establecida como una jerarquía de redes, y hará uso de la actual infraestructura, como el backbone NSFNET de la NSF, la ESNET del Departamento de Energía, la NSI de la NASA, y TWBNET del Departamento de Defensa!!.

\section{COSTA RICA Y SU INFRAESTRUC- TURA PARA REDES DE COMUNICACION COMPUT ARI- ZADAS}

Costa Rica, un pequeño país en el Itsmo Centroamericano, ha alcanzado niveles aceptables de desarrollo en algunos campos, como por ejemplo: educación, servicios de electricidad y telecomunicaciones, así como infraestructura computacional. Más aún, tales logros han sido generados bajo un sistema de democracia política, participación y consenso.

En este país, la acción del gobierno lo ha hecho protagonista relevante de los esfuerzos de desarrollo de infraestructura, distribuidos en toda su área geográfica. Las instituciones del gobierno también tienen instalado un alto porcentaje del parque computacional del país.

En la actualidad, bajo el enfoque mencionado en el apartado 11 ,el papel del gobierno consiste, no en desarrollar ventajas competitivas, sino en crear y mejorar las condiciones para que ellas sucedan!2. Entre tales condiciones están: un marco legal actualizado, el establecimiento de estándares de calidad, la inversión para investigación básica, y la regulación para adquirir y construcción de infraestructura. 
En 1985, el- Congreso abolió impuestos, a la importación de microcomputadoras. Hoy hay cerca de 50 personas por microcomputadora. El Programa de Informática Educativa de la Fundación Ornar Dengo, con el soporte del Ministerio de Educación Pública y del MIT, ha puesto herramientas computacionales de apoyo educativo, en casi $50 \%$ de todas las escuelas primarias del país, para fortalecer los procesos cognocitivos y la creatividad de los niños.

En 1988, se creó la "Comisión Nacional de Política Informática", con participación de las universidades, empresas relevantes en el área de la Telemática y el Gobierno

En forma creciente, en los últimos años, el sector privado y las universidades, con apoyo internacional, han contribuido también al desarrollo de infraestructura en el país, como por ejemplo, el desarrollo de las redes locales para Bitnet e Internet.

\section{LA UNIVERSIDAD DE COSTA RICA} (UCR): EL DESARROLLO DE UNA RED "CORPORATIVA" DE ALCANCE INTERNACIONAL: la UCRNet

En cualquier país de Centroamerica, una persona puede realizar una conexión personal a las redes internacionales, teniendo un código de acceso y pagando el costo de la llamada internacional. Algunas personas $\mathrm{u}$ organizaciones brindan este ipo de servicio local y limitado, al cual se le llama UUCP ("Unix to Unix Copy Program").

En noviembre de 1991, la Universidad de Costa Rica creó el primer acceso nacional a la red Bitnet, usando la infraestructura nacional de las mpresas ICE y RACSA. El "nodo", o máquina de comunicaciones y servicios de Bitnet, tuvo por nombre internacional UCRVM2.BITNET. Bitnet n Costa Rica llegó a contar con alrededor de 2000 ssuarios, de los cuales el $70 \%$ eran académicos o studiantes de la propia UCR, y los demás, isuarios de otras instituciones nacionales y entroamericanas. Panamá llegó a estar nterconectada a través de este nodo.
En febrero de 1993, Costa Rica se conectó a la red Internet, inicialmente a través de la Universidad de Costa Rica, usando la infraestructura nacional mencionada, a $64 \mathrm{Kbps}$ (Kilo bits por segundo). Durante el presente año esta velocidad podría aumentar a $128 \mathrm{Kbps}$ o más. En la Internet de Costa Rica existen varios nodos distribuidos por todo el país.

En Costa Rica, varias instituciones de enseñanza e-investigación se han interéonectado a Internet, formando la red académica nacional, llamada CRNet. Sin embargo, el servicio Internet se extiende a dos ramas adicionales: la de organizaciones privadas y la de instituciones públicas. Al presente, la Academia Nacional de Ciencias, junto con la Asociación CRNet son las organizaciones encargadas de administrar la asignación de direcciones a los nodos del país.

En la Universidad de Costa Rica se está construyendo una red telemática, principalmente para servicios docentes y de investigación, capaz de transportar voz, datos, sonido e imagen. Esta red tiene por nombre "Red Telemática de la Universidad de Costa Rica", conocida internacionalmente como UCRNet.

Esta red tiene un primer nivel, o "backbone", de fibra óptica para velocidades iniciales de 10 Mbps (millones de bits por segundo), con capacidad instalada para trasladarla a 100 Mbps en el corto plazo. Esta red enlazará las diferentes sedes, hospitales, institutos y centros de investigación que tiene la UCR dispersos en todo el país.

\section{CONCLUSION}

Ante los cambios globales mencionados, algunos países en desarrollo, aquellos con las condiciones históricas, culturales y de infraestructura adecuadas, tienen una "ventana histórica" que les puede permitir dar un "salto" desde su presente condición, hacia la creación de sus propias ventajas competitivas, realizadas por medio del recurso humano y la infraestructura localizada en su territorio. 
Las TI hacen posible el compartir con mayor eficiencia yen forma globalizante el conocimiento científico, tecnológico, comercial, etc. La competencia basada en el conocimiento no es un "juego de SUOlacro", porque este debe compartirse para que crezca. Por ello, un país como los EVA, con su capacidad líder en la producción de conocimiento e innovaciones, busca en las redes de comunicación, mejores formas de seleccionar y distribuir $^{13}$ información pertinente para su capacidad comercial, finaciera, etc., y por tanto, mejorar sus ventajas competitivas globales.

Las universidades deben incrementar sus capacidades internas para apoyar los procesos de transferencia, adaptación y generación de conocimientos científicos y tecnológicos. Las redes de comunicación las fortalecerán en sus procesos de comercialización de conocimientos y para desarrollar proyectos conjuntos con otras instituciones generadoras y transmisoras de conocimiento, así como con el sector productivo.

Las redes nacionales deben dedicarse tanto a las aplicaciones académicas "puras", como a otros tipos de usos, por ejemplo, los comerciales, y estar conectadas a la red de redes Internet, para apoyar el desarrollo económico de sus países

En parte, el éxito del concepto "Internet" consiste en la participación directa del usuario en su administración. Por ello, en los países en que se establezca un brazo de esta red, los usuarios deben tener participación activa y poder directo en su formación, administración y desarrollo .

\section{BIBLIOGRAFIA.}

La siguiente es la bibliografía usada en la preparación de este artículo:

- Aronson, Jonathan (1992). "Telecommunications Infrastructure and U.S. International Competitiveness". In, Annual Review of Institute for Information Studies. US, pp. $55-90$.

- Bifani, Paolo (1988). Property Rights. High Technology and International Trade. Geneva, Undp/UnctadlEclac.
- Branscomb, Lewis (1992). "U.S. Scientific and Technical Information Policy in the Context of a Diffusion-Oriented National Technology Policy". In, Government Publications Review. US, Vol 19, pp. 469 - 482.

- Cronin, Francis (1990). The Contribution ofthe Tele-communications Infrastructure to $\mathrm{Ag}_{-}$ gregate and Sectorial Efficiency. US, DRIl McGraw Hill, November.

- Gerber, Cheryl (1993). "Booming Commercial Use Changes Face of Internet". In, Info World. California, April12, pp. 1,38.

- Kahin, Brian (1993), editor. Building Information Infrastructure U.S, McGraw-Hill, Inc.

- Kahin, Brian (1991). "Information Policy and the Internet: Toward a Public Information Infrastructure in the United States". In, Government Publications Review. US, Vol. 18,pp. $451-472$.

-Levine, Jonathan (1993). "Wriston' sNew World". In, . $\quad$ US, March 29, p. 56.

- Office ofScience and Technology Policy (1992). The National Research and Education Network Program: A Report to Congress. Washington, D.C., Executive Office ofthe President, December.

- Porter, Michael (1990). The Competitive Advantage efNations. New York, The Free Press.

- Rapaport, Richard (1993). "Singapore. Still Onward and Upward?". In, Eorbes Asap. US, March 29, pp. $78-88$.

- Tassey, Gregory (1992). Technology Infrastructure and Competitive Position. Boston, Kluwer Academic Publishers.

- "Thinkers versus Dreamers" (1989). In, The Economist. December 23, pp. 87 - 88 .

- Weber, Alan (1993). "What's so New About the New Economy?". In, Harvard Business Review". USA, January - February, pp. 24-42. 